\title{
Editorial 2019 In Review
}

\section{Fred R. Volkmar ${ }^{1}$}

Published online: 2 May 2020

๑) Springer Science+Business Media, LLC, part of Springer Nature 2020
Continuing a decade-long trend, Journal submissions have increased steadily, year by year, from 740 in 2007 to 1,147 in 2019. Of the papers received by JADD last year, 462 were ultimately accepted - almost invariably following at least one revision. The associate editors and I were pleased to see the growth of scholarship on autism around the world; this past year authors from 73 countries submitted to the Journal. The downside of the increased number of submissions is that our standards are necessarily going to become stringent. With the increased number of submissions we are increasingly aware of the problem of 'reviewer fatigue,' and the associate editors and I are increasingly going to either reject papers without review or redirect them to a different Journal. Review articles are now routinely redirected to our companion Journal, Review Journal of Autism and Developmental Disorders, or elsewhere.

As in the past we strive to make the review process as efficient as possible. Once a paper has cleared the initial internal review for adherence to journal style, the average time from first submission to initial decision is just over 6 weeks. This reflects the diligence of the journal office in helping insure papers are ready for review and the responsiveness of our reviewers. Occasionally a paper on a complex or highly focused topic can require a lengthier review time; in these cases, the Journal appreciates authors' patience in awaiting feedback.

It is important for authors to remember that once a paper has been accepted the pace of electronic publication (complete with a doi) is very rapid so it becomes even more important that submissions be in final form without error when they go out to review. The JADD office staff checks for some basic things like format before papers come to me for assignment but they cannot detect errors of scholarship - hopefully (but not always) these are noted in review. In about one-third of cases, authors are asked

Fred R. Volkmar

fred.volkmar@yale.edu

1 Yale University, New Haven, CT, USA to make formatting corrections before they come to peer review. We also remind authors that duplicate publication is not allowed and authors certify this to us as part of the submission process along with ethical research conduct and so forth. Springer Nature is a member of COPE, the Committee on Publication Ethics, and the COPE guidelines are followed for all research integrity issues that come up in relation to JADD articles both under review and published.

The Journal continues to expand its editorial board and add Associate Editors as members rotate off. The Journal would like to extend a warm welcome to our newest Associate Editors: Cara Pugliese, Ph.D. and Shefali Spurling Jeste, M.D. We bid a fond farewell to the following board members: Nick Chown, Independent Researcher, Annilies de Bildt, Ph.D., Iliana Magiati, Ph.D., D.Clin.Psy., Nancy Minshew, M.D., Laura Morett, Ph.D., Matthew Mosconi, Ph.D.,

Deborah Riby, Ph.D., Susan White, Ph.D. Each has generously and diligently supported the Journal's mission.

The Journal is deeply grateful for the continued dedication of the remaining Associate Editors: Alice S. Carter, Ph.D., Craig A. Erickson, M.D., Carla Mazefsky, Ph.D., Christopher J. McDougle, M.D., James C. McPartland, Ph.D., Patricia A. Prelock, Ph.D., Brian Reichow, Ph.D., Hanna Stevens, M.D., Ph.D., Gerrit Ian Van Schalkwyk, M.D., Giacomo Vivanti, Ph.D., and Marc Woodbury-Smith, $\mathrm{PhD}$, MRCPsych.

We welcome new editorial board members including Yusuf Akemoglu, Ph.D., Angelica Marie Astor, Ph.D., Erinn Nell Barry, M.A., Mark Brosnan, Ph.D., Alastir Craig Clarkson, MSc, Daniel Dunleavy, MSW, Ph.D., Rebecca Elias, Ph.D., Erin Kang, M.A., Mr. Kenneth Larsen, Rachel Loftin, Ph.D., Michael J. Morrier, Ph.D., BCBA-D, and Luigi Mazzone, M.D., Anders Nordahl-Hansen, Ph.D., Justin Rowberry, M.D., Jennifer Lawson Stapel-Wax, PsyD, and Rutger Jan van der Gaag, M.D., Ph.D.

The associate editors, office staff and I also extend our very best wishes and many thanks to Judy Jones and Michelle Tam for all their hard work and support over the years and we welcome Jennifer Hadley as Senior Editor and Paola Yuli as Editorial Assistant. 
We continue to encourage authors to use the option of online publication of supplementary material as "Extra Supplementary Material" (ESM). As noted in previous editorials, JADD typically does not accept review papers, which are typically referred to Springer's companion journal, the Review Journal of Autism and Developmental Disorders; however, the Journal continues to be interested in metaanalyses. In addition, JADD accepts case report submissions only in the form of letters to the editor; case studies may be submitted as standard "original research" papers.

The Journal continues to encourage, when possible, the grouping of papers on related topics in special issues/sections. We currently have three of these now in preparation - one on early detection, another on suicidality and selfharm, and one looking at progress in autism work in a range of areas 40 years after the publication of DSM-III. Consistent with Journal policy, all papers submitted to special sections/issues are subject to the standard double-blinded peer-review process as other papers.

In the Journal office, Lori Klein, Editorial Manager, and Monica Mleczek, Editorial Assistant, work diligently to ensure that every submission conforms to $J A D D$ standards before it enters peer review, thereby streamlining the process for the Editor and Associate Editors.

Listed in the following section are the names of the individuals who have contributed one or more reviews to the Journal this year. Although far from perfect, peer review remains the most effective way for the field to ensure the quality of the scholarly product. $J A D D$ thanks these individuals for their hard work and dedication.
Adriana Barbosa Gonasalves

Ingela aahslund

Mohammad Hadi Aarabi

Heidi Abadeh

Véronique Abadie

Kanakalatha Abbagani

Leonard Abbeduto

Kirsten Abbot-Smith

Hadeer Abd El-Khalik

Morsi Abdallah

Sarah Abdallah

Nabila Abdelmeguid

Maha Abdelmohsen

Emily Abel

Kathryn Abel

Nadia Abouzeid

Gregory Abowd

Mark Abrams

Michael Absoud

Amina Abubakar

Sana Abu-Dahab

Kamal Abulebda

Amy Accardo

Pasquale Accardo

Louise Acker

Maria Acosta

Catherine Adams

Daniel Adams

Dawn Adams

Denise Adams

Kenneth Adams

Lynn Adams

Nena Adams

Sherri Adams

Lucy Adamson
Andrew Adesman

Noga Adler-Jacob

Ralph Adolphs

Francisca aerts

Saskia Aerts

J'Anne Affeld

Eileen Africa

Soheil Afshar

Bahareh Afsharnejad

Alireza Aghaz

John Agnew

Sandra Maria Herondina Coelho

Aguiar

Nadia Aguillon-Hernandez

Belhadj Ahlem

Noelle Ahlert

Ahmad Ahmadi

Alex Ahmed

Asif Ahmed

Deka Ahmed

Shaf Ahmed

Si-Nae Ahn

Ingela Åhslund

Banu Ahtam

Deepti Ahuja

Yog Ahuja

Kenneth Aitken

Alice Ajanga

Zahra Akbari Bayatiani

Ekrem Akbug

Murat Akcakaya

Hironori Akechi

Burcu Semin Akel

Yusuf Akemoglu

Jessica Akers
Leyla Akoury Dirani

Selin Aktan Guloksuz

Nebal Al Anbar

Mohammad Aladawi

Michele Alaniz

Erna Alant

Omar Alawajee

Laila AL-Ayadhi

Rachel Albone-Bushnell

Lilia Albores-Gallo

Matthew Albrecht

Jose Alcántara

José Ignacio Alcántara

Alyssa Alcorn

Megan Alder

Ben Alderson-Day

Catherine Aldred

Fiona Aldridge

Anhaar Al-Enezi

Michael Alessandri

Silvia Alessi-Severini

Abigail Alexander

Lisa Alexander

Basmah Alfageh

Omar Al-Farsi

Yahya Al-Farsi

Eglal Ali

Shaimaa Al-Kandari

Jamal Alkhateeb

Clare Allely

David Allen

Greg Allen

Harriet Allen

Melissa Allen 
Micah Allen

Richard Allen

Sarah Allen

Hiie Allik

Carrie Allison

Tracy Alloway

Watfa AL-Mamari

Sarah al-mazidi

Joana Almeida

Telma Almeida

Fahad Alnemary

Faisal Alnemary

Hanna Alonim

Ragea Alqahtani

Norah Alrusayni

Ali Alsaad

Rehab Alsaedi

Ayten Alsaffar

AHMAD AL-SAGARAT

Majd Al-Soleiti

Mohammad Altamimi

Mareike Altgassen

Matthew Altiere

Eric Altschuler

Gianpaolo Alvari

Charlotte Alverson

Ashwaq Alzamel

Nouf Alzrayer

MICHAEL AMAN

Lais Amaral

Fiona Ambery

Ben Ambridge

Katarina Ament

Catherine Ames

Lorene Amet

Sanjiv Amin

F. Amirabdollahian

Letícia Amorim

R. Amoss

Miguel Ampuero

Alok Amraotkar

Dima Amso

Evdokia Anagnostou

Lata Ananthan

Emily Anderberg

Allan Andersen

Per Normann Andersen

Anastasia Anderson

Angelika Anderson

Connie Anderson

David Anderson

George Anderson

Karyn Kai Anderson
Lori Anderson

Sophie Anderson

Gunilla Andersson

Clara Andrés-Roqueta

Karen Ang

Amber Angell

Kathleen Angkustsiri

Manya Angley

Julia Anixt

Pamela Anketell

D. Annaz

Photios Anninos

Sophie Anns

Ligia Antezana

Laura Gutermuth Anthony

Kevin Antshel

Yuta Aoki

Fabio Apicella

Kaija Appelqvist-Schmidlechner

Manijheh Arabi

Madlena Arakelyan

Weerayot Aramphianlert

Adi Aran

Eva Araujo

Emily Arcia

Doreen Arcus

Araceli Arellano

Pablo Argibay

Malena Argumedes

A. Ariheiam

Jodie Armstrong

Rebecca Armstrong

Arvin Kim Arnilla

Samuel Arnold

TAIBA AROOJ

Manish Arora

Narendra Arora

Sudha Arunachalam

Kosuke Asada

Kristie Asaro-Saddler

Jill Ashburner

Manzar Ashtari

Chris Ashwin

Karen Ashwood

Paul Ashwood

Sorayya Askari

Kathleen Aspiranti

Michal Assaf

Susan G. Assouline

Gray Atherton

Anthony Atkinson

Hjördís Atladóttir

Tony Attwood
Adham Atyabi

Rachel Hoi Yan Au

Terry Au

Benoite Aubé

Melinda Jones Ault

Karla Ausderau

Elizabeth Austin

Jillian Austin

Bonnie Auyeung

Keren Avirame

Einat Avni

Michal Avrech Bar

Elizabeth Aylward

Izza Ayub

Alvi Azad

Amy Azano

Nadia Azar

Samy Azer

Fatemeh Azimi Amoli

Aley Babb

JOCELYNE BACHEVALIER

Christian Bachmann

Elisa Back

Michelle Backley

Elizabeth Bacon

Andrzej Bączkowski

Johanna Badcock

Stephanie Bader

Young Seh Bae

Diana Baekey

Carolina Baeza-Velasco

Amaria BAGHDADLI

Christopher Baglio

Daniel Bagner

Deena Bahadur

Eva Baharav

Fatimah Bahrami

Jeremy Bailenson

Anthony Bailey

Benjamin Bailey

Donald Bailey

Sacha Bailey

Gillian Baird

Ayelet Baisa

Inmaculada Baixauli Fortea

Dana Lee Baker

Jason Baker

Kimberly Baker

Sara Baker

Amanda Bakian

Trine Lise Bakken

Theo Bakker

Asmaa Bakroon 
Elgiz Bal

Marjolijn Bal

Vanessa Bal

Giulia Balboni

Lindsey Balderaz

Eleni Baldimtsi

Susanna Baldwin

Michelle Ballan

Carrie Ballantyne

Jaime Ballard

Elizabeth Ballinger

Joshua Balsters

Linda Bambara

Stacie Bancroft

Cláudia Bandeira de Lima

Linda Bandini

Arup Bandyopadhyay

Janet Bang

Sanja Banjavcic

John Bankart

Rahul Bansal

Vanessa Bao

Wei Bao

Patricia Baptista

Urszula Barańczuk

Grace T. Baranek

William Barbaresi

Josephine Barbaro

Angela Barber

Olivia Barber

Pietro Barbetta

Gardenia Barbosa

Adriana Barbosa- Gonçalves

Alejandra Barcala

Cécile Bardon

Evelien Barendse

Brian Barger

Sarah Bargiela

Erin T. Barker

Gregory Barnes

Julia Barnes

Petra Barneveld

Erin Barney

Kelly Barnhill

L Jarrett BARNHILL

Marie-Pierre Baron

SIMON BARON COHEN

Amy Barrett

Daniel Barron

Patricia Barros

Colleen Barry

Erinn Barry

Tammy Barry
Lawrence Bartak

Catherine Barthelemy

James Bartok

James Bartolotti

Erin Barton

Marianne Barton

Andrea Barton-Hulsey

Emanuele Basile

Laura Bassette

Nico Bast

Amy Bastian

Agatino Battaglia

Giuseppe Battaglia

Sema Batu

Debra Bauder

Lori Baudino

Kim Bauerly

Sarah Baum

Melissa Bauman

Nirit Bauminger-Zviely

Edith Bavin

Mojdeh Bayat

Nadia Bayou

Allison Bean

Jessica Bean

KIMBERLY BEAN

Peter Bearman

Karen Bearss

Elliott Beaton

Myriam Beauchamp

Sylvie Beauchamp

Jessica Beauvais

James M. Bebko

Tracy Becerra-Culqui

Kelly Beck

Joanna Beckett

Jennifer Beers

Sander Begeer

Jannath Begum Ali

Rebecca Beights

Esubalew Bekele

Maria Bella

Alessio Bellato

Giulia Bellesi

Scott Bellini

Katherine Bellone

Matthew Belmonte

Dafna Ben Bashat

Dorit ben Shalom

Mohamed Abdelkader Bencherif

Konabe Bene

Teal Benevides

Fabiana Benites
Esther Ben-Itzchak

Emily Bennett

Kyle Bennett

Marc Bennett

Matthew Bennett

Patrick Bennett

Teresa Bennett

Loisa Bennetto

Stephen Benning

Ayelet Ben-Sasson

Paul R. Benson

Rhianna Benson

Valerie Benson

Catherine Bent

Satoshi Beppu

Carmen Berenguer

Kristin Berg

Itai Berger

Natalie Berger

Kathleen Bergman

Nils Bergman

Thomas Bergmann

Märith Bergström-Isacsson

Lauren Berkovits

Steven Berkowitz

Fabrice Berna

Paola Bernabei

Thomas Berney

Raphael Bernier

Virginia Berninger

Jon Bernstein

Dominique Béroule

Kari Berquist

David Berry

Leandra Berry

Colleen Berryessa

Elizabeth Berry-Kravis

Denise Berte

Sylvie Berthoz

Armando Bertone

Jo Bervoets

Catherine Best

Sara Best

Terrence Bethea

Alison Betz

Karin Beuker

David Beversdorf

Zubin Bhagwagar

Anjana Bhat

Anjali Bhatara

David Bickar

Eric Bieniek

Thierry Bienvenu 
Elizabeth Biggs

Lucy Bilaver

Deborah Bilder

Niels Bilenberg

Lucia Billeci

Vincent Billock

Eva Billstedt

Tracy Bingham

Marleen Bink

Federico Bionda

Geoff Bird

Geoffrey Bird

E. Birmingham

Monica Biscaldi

Dorothy Bishop

Haley Bishop

Somer Bishop

Somer L. Bishop

Lauren Bishop-Fitzpatrick

John Bissonnette

Vicki Bitsika

Jan Blacher

Melissa Black

Ksusha Blacklock

Abigail Blackman

A. Josephine Blagrave

Kwang-Sun Blair

Erik Blaser

Lisa Blaskey

Gene Blatt

Hanie Blenner

Els Blijd-Hoogewys

Michael Bloch

Aaron Blocher-Rubin

My Blomwvist

Sarah Bloom

Luke Bloy

Wendy Blumenthal

Jin Bo

Leticia Boada

Allira Boadle

Jonna Bobzien

Anne Böckler

JAMES W BODFISH

Doug Bodin

Stefanie Bodison

Kimberly Bodner

Bart Boets

Hans Bogte

Gal Bohadana

Irina Boksha

Francois Bolduc

Vedrana Bolic Baric
Sven Bolte

Patrick Bolton

Daniel Bone

Mortaza Bonyadi

Susan Bookheimer

Anne Boomsma

Hannah Boonen

Rhonda Booth

Tom Booth

Karen Bopp

emre bora

Elise Borah

Marta Borgi

Ross Borland

Carrie Borrero

Dienke Bos

Marieke Bos

Marianna Boso

Goele Bossaert

Kristen Bottema-Beutel

James N. Bouder

Kelsie Boulton

Thomas Bourgeron

Helen Bourke-Taylor

Sarra Bouslah

Rose-Mary Boustany

Henrietta Bowden-Jones

Katherine Bowers

Dermot M. Bowler

Susan Bowyer

Brian Boyd

LouAnne Boyd

Megan Boyle

Wiebe Braam

Catherine Bradley

Jessica Bradshaw

John Bradshaw

Lauren Bradstreet

Danielle Brady

Dannielle Brady

Nancy Brady

Nuala Brady

Meghan Brahm

Kira Branch

Harrison Brand

Cinara Brandão de Mattos

Alice Brandwein

James Brasic

Claude Braun

$\mathrm{M}^{\mathrm{a}}$ Ángeles Bravo Álvarez

Signe Bray

Joel Bregman

Emily Bremer
Avril Brereton

Alexis Brewe

Neil Brewer

Rebecca Brewer

Rachel Brezis

Jessica A. Brian

Sarah Brieber

Mary Briggs

Amanda Brignell

Anita Brito de Jesus

Matthew Brock

Karen Brockland

Sarabeth Broder-Fingert

Neil Broderick

Neill Broderick

Briana Bronstein

Lauren Brookman-Frazee

Johnell Brooks

Mark Brosnan

Leslie Bross

Diane Browder

Alan Brown

Alec Brown

Amy Brown

Benjamin Brown

Caroline Brown

Heather Brown

Jane Brown

Ted Brown

Charlotte Brownlow

Jennifer Bruno

Victoria Brunsdon

Anat Brunstein Klomek

Lauren Bryant

Susan Bryson

maria pia bucci

Susanne Buchmayer

Catelyn Buck

Dejan Budimirovic

Shaimaa Bugshan

Jan K. Buitelaar

Daniela Bulgarelli

Jennifer Bullen

Myra Beth Bundy

JACOB Burack

Sloane Burgess

Joshua Burk

Meghan Burke

Shanna Burke

Karen Burkett

Carolyn Burkhart

Courtney Burnette

Priscilla Burnham Riosa 
Amy Burns

Claire Burns

Jan Burns

Teresa Burrell

Catherine Burrows

David Burt

Jenny Burton

Simon Bury

Hillary Bush

Paige Bussanich

Merlin G. Butler

David Buttelmann

Eric Butter

Kaitlyn Butterfield

Felicity Butterly

Thomas Butterworth

Agnieszka Butwicka

Caroline Buzanko

E. Sandra Byers

Breanne Byiers

Mitchell Byrne

Anthony Cacace

Jessica Cadette

Collette Caffrey

Eilidh Cage

Ru Ying Cai

Sara Calderoni

Federico Calegari

Nathan Call

Kevin Callahan

Louis Camilleri

Amy Camodeca

Colin Campbell

Daniel Campbell

Jonathan Campbell

Kathleen Campbell

Martha Karen Campbell

Susan Campbell

Giovanna Cristina Campione

Ricardo Canal-Bedia

Josefa Canals Sans

Fatih Canan

MICHELA CANDINI

Molly Candon

John Cannell

Helen Cannella-Malone

Catherine M. Capio

Barbara Caplan

Mary Catherine Cappadocia

Nicole Capriola-Hall

Kelly Caravella

Paul Carbone

V.J. Carbone
Teresa Cardon

Cláudia Cardoso-Martins

Sarah Carlon

Derek Carlson

Emilia Carlsson

Joana Carmo

Montserrat Caro

Carlos Carona

Kimberly Carpenter

Laura Carpenter

Malinda Carpenter

Barbara Carpita

Staci E Carr

Themba Carr

Arnaud Carré

Sarah Carrington

CRISTINA CARROZZA

Tana Carson

Alice Carter

Erik Carter

Virginia Carter Leno

Gemma Carvill

Maria Casagrande

Michael Casale

Manuel Casanova

Paul Cascella

Carissa Cascio

Devin Casenhiser

Jane Case-Smith

Cara Cashon

Antonio Cassella

Sarah Cassidy

Ana Castejon

Miguel Castelo-Branco

George Castle

Kamila Castro

Linda C. Caterino

James Cauraugh

Paul Cavanagh

Homero Cavazos

Fabienne Cazalis

Hellas Cena

Carolina Cerezo

Leonardo Cerliani

Sharon Cermak

Paige Cervantes

Virginia Chaidez

Kusalaporn Chaiudomsom

Bhismadev Chakrabarti

Anne Chalfant

Brigitte Chamak

Stephanie Chambaron-Ginhac

Angela Chamberlain
Mary Chambers

Thierry Chaminade

Agnes Chan

John Chan

Kevin K. S. Chan

Meingold Hiu-ming Chan

Victoria Chan

Vivien Chan

Wai Chan

Robert Chandler

Bryant Chang

Fong-Ching Chang

Hsueh-ling chang

Ya-Chih Chang

Leigh Chapman

Geneviève Charbonneau

Marjorie Charlop

Anna Charlton

Shawn R. Charlton

Tony Charman

Gregory Chasson

Lilian Chateau

Alfred Chavira

Katarzyna Chawarska

Nancy Cheak-Zamora

Derserri Chee

Briella Chen

Chia-Hsiang Chen

Chien-Hsu Chen

Fu-Chen Chen

Hao Chen

Huaihou Chen

Jing Chen

Kuan-Lin Chen

Lei-Shih Chen

Lianjun Chen

Li-Chiou Chen

Nigel Chen

Szu-Han Chen

$\mathrm{Xi}$ Chen

Yu-Wei Chen

Karen Chenausky

Chia-Hsiung Cheng

K Cheon

Ludmila Cherenkova

Keely Cheslack-Postava

Steven Chesnut

Candice Chi-Hang Cheung

Mei-chun Cheung

Coralie Chevallier

Donald Chi

Chung-Hsin Chiang

Hsu-Min Chiang 
Flavia Chiarotti

Sarina Hui-Lin Chien

Yiling Chien

Deb Childress

Iris Chin

Meia Chita-Tegmark

Morgan Chitiyo

Sepideh Chitsaz

Suk Ling Karoline Chiu

Colby Chlebowski

Margaret Chojak

Weerasak Chonchaiya

Ivy Chong

Walid Choucha

Roula Choueiri

Brea Chouinard

Philippe Chouinard

Kamalika Chowdhury

Nicholas Chown

Shawn Christ

Lisa Christensen

Jens Christiansen

Sheila Christopher

Jie-Yu Chuang

Kyong Mee Chung

Moo Chung

Peter Chung

Wendy Chung

Franceli Cibrian

DOMENIC CICCHETTI

Zuleyha Cidav

Joseph Cihon

Federica Cilia

Jacqueline Cintra Santos

Remzi Oğulcan ÇIRAY

Krista Clancy

Emily D. Clapham

Isabel Clare

Carol J. Clark

Karen Clark

Christopher Clarke

Alastair Clarkson

Joanne Cleland

Caitlin Clements

Caroline Clements

Tess Clifford

Yeaton Clifton

Robert Coben

Virginie Cobigo

Michael Cocchiola

David Cochran

Emily Coderre

M. JUSTIN COFFEY
Marika Coffman

Truman Coggins

Ira Cohen

Jeremy Cohen

Shana Cohen

Gina Colaizzo

Marilyn Coleman

Edmund Coleman-Fountain

Livia Colle

Simon Collinson

PAOLA COLLOZO

Barbara Colombo

Andrew Colombo-Dougovito

Drew Coman

Eric Common

Emma Condy

Olivia Conlon

Carmel Conn

Caitlin Conner

Annemarie Connor

MAUREEN CONROY

Massimiliano Conson

John Constantino

Anne Cook

Barbara Cook

Jennifer Cook

Kathy Cook

Lauren Cook

Megan Cook

Richard Cook

Frederick Coolidge

Kelly Coons

Miriam Cooper

Rose Cooper

Ruth Cooper

Christine Cooper-Vince

Blythe A. Corbett

Lisa Cordeiro

Christina Cordero

Reinie Cordier

Karly Cordova

Carlos Córdova-Fletes

Phil Corlett

K F Magnus Cormack

Laura Corona

Natália Correia

Maria Jose Cortes

Flavia Cortesi

Joanna Cosbey

Andreia Costa

Hélène Cottenceau

Kristina Cottle Feldman

Albert Cotugno
Elizabeth Couchon

ERIC COURCHESNE

Daniel Coury

Marieke Coussens

Romain Coutelle

Melanie Couture

Hilary Cowan

Richard Cowan

Bradley Cox

Daniel Cox

David Cox

Stephany Cox

Phyllis Coyne

Patrick Craanen

Lisa Crabtree

Daina Crafa

Jaime Craig

Elizabeth Ruth Crais

Madison Crandall

James Crane

Laura Crane

Jewel Crasta

Susan Crawford

Ann Creaby-Attwood

MARGARET CREEDON

Elizabeth Cridland

Alessandro Crippa

Lisa A. Croen

Pamela Crooke

Elizabeth Cross

Liam Cross

Morgan Crossman

Rachel Crowe

Ciera Crowell

Judith Crowell

Shannon Crozier

Lidia Cruz (do not invite)

Elisa Cruz-Torres

John Cryan

Laura Culshaw

Janet Cummings

Ali Cunningham

Amber Cunningham

William Cunningham

Michelle Curran

Carol Curtin

Luke Curtis

Sarah Curtiss

Elizabeth Curtiss-Cabell

Helio Clemente Cuve

Hanna Cygan

Kathryn Czepiel

Nikko S. Da Paz 
Dave Dagnan

Svenolof Dahlgren

Yael Dai

Dina Dajani

Andrew Dakopolos

Brittany Dale

Elaina DaLomba

Kirsten Dalrymple

Cara Damiano

Jesper Dammeyer

Coral Dando

Amy Daniels

Julie L. Daniels

Judith Danovitch

Laura D'Antuono

Nidal Daou

Mirella Dapretto

Ahmad Daryani

Gail Daumit

Hayley Dauterman

Roeliena Davids

Bridget Davidson

Larry Davidson

Meghan Davidson

Alison Davies

Cynthia Davis

Greg Davis

Jennifer Davis

Jolin Davis

Naomi Davis

Paige Davis

Rachael Davis

Thompson Davis

Tonya Davis

Leann DaWalt

Tamara Dawkins

Geraldine Dawson

Paul Dawson

Jessica Day

Taylor Day

Derek De Beurs

Annelies de Bildt

Beth De Grace

Maretha V. de Jonge

Berenice de la Cruz

Ashley de Marchena

Cristiane de Paula

Sarah De Pauw

Silvia De Rubeis

Caroline de Theije

Danielle de Veld

Annelou de Vries

Marieke de Vries
Petrus de Vries

C.F. De Winter

Kim Dean

Michelle Dean

Kyle Deane

Anders Dechsling

Deborah DeCloedt Pincon

Thomas DeGeorge

Linda Dekker

Vera Dekker

Laura Dekkers

ARACELI DEL POZO ARMENTIA

Jonathan Delafield-Butt

Jennifer Delahaye

Monica Delano

Florine Dellapiazza

Kristin Dell'Armo

Sonja Delmonte

Malika Delobel-Ayoub

Caroline Demily

Şenol Demirtaş

Carly Demopoulos

Allison Dempsey

Jack Dempsey

Jacquiline den Houting

Danielle DeNigris

Kristina Denisova

Katie Denman

Tanya Denmark

Barbara D'Entremont

Tiffany Denyer

Kathy DeOrnellas

Lesley Deprey

Cyrielle Derguy

Aaron R. Deris

Melissa DeRosier

Christine Deruelle

Pushpal Desarkar

Peter Deschamps

MC DeSoto

Whitney Detar

Stephen I. Deutsch

Elisabeth DeVilbiss

Deborah Dewey

Jeroen Dewinter

Jessica DeWitt

Katie Dhingra

Adriana Di Martino

Agnese Di Napoli

Magda Di Renzo

Briano Di Rezze

Gary Diamond

Thiago Dias
Gabriel Dichter

Abigail Dickinson

Chata Dickson

Kelsey Dickson

André Diedrich

Joshua Diehl

Lisa Dieleman

claudine dietz

T. Diggle

Renee Dijkhuis

Ibrahim Diken

Lisa Dille

Karola Dillenburger

Adriana DiMartino

Adele Dimian

Dimitrios Dimitriou

Nevena Dimitrova

Helen Ding

Ilan Dinstein

Eric Dion

Genevieve Dion

Cheryl Dissanayake

Charlotte DiStefano

Dennis Dixon

Mark Dixon

Aleksandra Djukic

Phuong Do

Karen Dobkins

Helen Dodd

Robin Dodds

Peter Doehring

Gwyneth Doherty-Sneddon

Hirokazu Doi

Smadar Dolev

Chrystina Anna Dolyniuk

Sarah C. Domire

Franc Donkers

Albert Donnay

David Donnelly

Brian D'Onofrio

Kathy Doody

Osama Dorgham

Michail Doumas

Katerina Dounavi

Jenny Downs

PETER W. DOWRICK

Amy Drahota

Daniel Drake

Jennifer Drake

Cathi Draper Rodriguez

Thomas Dratsch

Doniel Drazin

Meredith Dreyer Gillette 
Barbara Dritschel

Bradley Drysdale

Rennan Yanlin Du

Michaela DuBay

Indu Dubey

Ashley Dubin

Jessica Dudek

Katerina Dudley

Iva Dudova

Ana Dueñas

Emma Duerden

Ellen Duifhuis

Eric Duku

Claire Dumont

Thyde Dumont-Mathieu

Amie Duncan

Daniel Dunleavy

David Dunn

Winnie Dunn

CARL DUNST

V. Mark Durand

Maureen Durkin

Stephanie Durrleman

Anuradha Dutt

Susanne Duvall

Jorieke Duvekot

Karine Duvignau

Katharina Dworzynski

Tina Taylor Dyches

Kathleen Dyer

Elisabeth M Dykens

Kristy Dykshoorn

Jessica Dykstra Steinbrenner

David Dyment

Isabel Dziobek

Shaun M. Eack

Rita Eagle

Valsamma Eapen

Daniel Earixson

Karen Eastman

Linda C. Eaves

Christine Ecker

Andreas Eckert

Stephen Edelson

J. Christopher Edgar

Jamie Edgin

Andrea Edlow

E Edmiston

Sarah Edmunds

Amelia Edwards

Laura Edwards

Vincent Egan

Andrew Egel
Jeffrey Eggleston

Felicitas Ehlen

Jill Ehrenreich-May

Diane Ehrensaft

Inge-Marie Eigsti

Sigal Eilat-Adar

Stewart Einfeld

Abbey Eisenhower

Naomi Ekas

Leif Ekblad

Hanna Eklund

Farah El Zein

Sigmund Eldevik

Paul El-Fishawy

Rebecca Elias

Jed Elison

Susan Ellis Weismer

Mayada Elsabbagh

Sara Eltyeb

Junita Elvira

Marie Elwin

Leonardo Emberti Gialloreti

David Emerich

Eric Emerson

Natacha Emerson

Elisabeth Engberg-Pedersen

Karen Engel

Michael English

Peter Enticott

Sumari Erasmus

Gillian Erhabor

Annette Erlangsen

Anna Esbensen

Giovana Escobal

Esad Esgin

Karen Goldrich Eskow

Amy Esler

Samauneh Esmaeili

Joan Esse Wilson

Annette Estes

Angeles Estévez

Benito Estrada

beverley evans

E. Whitney Evans

Kris Evers

Joshua Ewen

Louise Ewing

Darryl Eyles

Maddalena Fabbri-Destro

Marc Fabri

Reina Factor

Charles Fage

Susan Faja
Terje Falck-Ytter

Ruth Falco

Dean Falk

Nicholas Falk

Marita Falkmer

Torbjorn Falkmer

Jin Fan

Huda Farah

Cristan Farmer

Teresa Farroni

Faraz Farzin

Daniel Faso

Aviva Fattal-Valevski

Víctor Faundes

Kate Favot

Kathleen Feeley

Bronwyn Fees

Deborah Fein

Carmen Feldhaus

Jacob Feldman

Maurice Feldman

Melanie Feldman

Ruth Feldman

Jennifer Felger

Christian Fellner

SARAH NINAN FENN

Rachel Fenning

Alana Fenton

Bradley Ferguson

Emily Ferguson

Jenny Ferguson

Ane Fernald

Sherira Fernandes

Thomas Fernandez

Samuel Fernandez-Carriba

Elisabeth Fernell

Suay i Lerma Ferran

Fernanda Ferreira

Angel Fettig

Juliana Fialho

Charlotte Field

TIFFANY FIELD

Joanne Fielding

Margarida Figueiredo-Braga

Marisa Filipe

PAULINE FILIPEK

Liora Findler

Jodene Fine

Jonathan Fine

Erinn Finke

Elizabeth Finnegan

Brenda Finucane

Chiara Fiorentini 
Angela Fish

Celia Fisher

Julia Fisher

Marisa Fisher

Inna Fishman

Kate Fiske Massey

Allison Fitch

Emily Fitzgerald

Jacqueline Fitzgerald

Michael Fitzgerald

Paula Fitzpatrick

Zoe Flack

Fay Fletcher

Sue Fletcher-Watson

Amanda Fleury

Veronica Fleury

Michelle Flippin

Margaret Flores

Dorothea Floris

Michael Flory

Rebecca Flower

Rachel Flynn

Jill C. Fodstad

Eric Fombonne

Trisha Forbes

Michelle Forcier

Talitha Ford

Baudouin Forgeot d'Arc

Eva Forman

Bonny Forrest

Cara Fosdick

Jennifer Foss-Feig

Kimberly Fournier

Elaine Fox

Martina Franchini

Grace Francis

KONSTANTINOS FRANCIS

Alcantud-Marín Francisco

Sophia Frangou

Frederick Frankel

Kirsten Frantzen

Lauren Franz

Jean Frazier

Thomas Frazier

Stephanny Freeman

Megan Freeth

Christine Freitag

Critsiane Freitas

Adel Fridhi

Eron Friedlander

Nora Friedman

Derrick Fries

UTA FRITH
Amy Fritsch

Odette Fründt

Richard Frye

Mitch Fryling

Toru Fujioka

Elizabeth Fuller

Kimberly Fuller

Natalia FUMAGALLI

Allyson Funamoto

Lawrence Fung

Joseph Furman

Laura Fusar-Poli

Avia Fux-Noy

Emily Fyfe

Lidia Gabis

Robin Gabriels

Terisa Gabrielsen

Daniel Gadke

Kenneth Gadow

Nicholas Gage

Sebastian B. Gaigg

Eynat Gal

Bobbie GALLAGHER

Louise Gallagher

Elena Gallitto

James Galloway

Jennifer Gallup

Devon Gangi

Kerstin Ganglmayer

Alexander Gantman

J. B. Ganz

Jeanette Garcia

OMAR GARCIA-LIEVANOS

Teresa Garcia-Pastor

Patricia García-Primo

H. Gardener

Emily Gardiner

Lauren Gardner

Sarah Garfinkel

Belinda Gargaro

John Jay Gargus

Marie-Ève Garon

Michael Garrett

Elena Garrido Gaitan

Marco Garzitto

Holly Gastgeb

Gordon Gates

Susan Shur-Fen Gau

Valerie Gaus

Line Gebauer

Cheryl Geisthardt

Nicholas Gelbar

Daniel Geller
Grace Gengoux

Stephen Gentles

Betholyn Gentry

Rita George

S Thomas George

Stelios Georgiades

Alexandra Georgiou

Bruno Gepner

Alan Gerber

Peter Gerhardt

Esther Germain

Monique Germone

Morton GERNSBACHER

Darios Getahun

Nancy Getchell

Elizabeth Getzel

Hilde M. Geurts

Tali Gev

Cindy Gevarter

Yasser Ghanbari

Mohammad Ghaziuddin

Laura Ghirardi@ki.se

DEBASRUTI GHOSH

SANJIB GHOSH

Subharati Ghosh

Katingo Giannoulis

Ellen Giarelli

Ellen Giarellli

Gofon Gibb

Varleisha Gibbs

Vicki Gibbs

Amelia Gibson

Sandrine GIL

Kimberly Gilbert

lina gilic

Carina Gillberg

Christopher Gillberg

Kristen Gillespie-Lynch

Whitney Gilliland

Jennifer Gillis

Alinda Gillott

Maria F. Gilmour

Nicole Ginn

Esther Ginsberg

Susan Girdler

Ivy Giserman-Kiss

Beth Glasberg

Emma Glasson

Melanie Glenwright

Derek Glidden

Laraine Glidden

Magdalena Glod

Ken Gobbo 
Linda Goddard

Leandra Godoy

Lakshmi Gogate

Eyleen Goh

Kwang Leng Goh

Tze Jui Goh

Ofer Golan

Rinat Gold

Melissa Goldberg

Rachel Goldin

Samantha Goldman

Sylvie Goldman

Janice Goldschmidt

Tina Goldsmith

Tejas Golhar

Amir Golriz

Alice Gomez

Esperanza Gómez Durán

Marie Gomot

Keiko Gondo

Anna Gonsiorowski

Corentin Gonthier

Ramon Gonzalez

Ana Maria Gonzalez-Barrero

Susan Goode

Charls Goodsel

Anthony Goodwin

Matthew Goodwin

Matthew S. Goodwin

Anuraag Gopaluni

Andrew Gordon

Iris Gordon

Phil Gorrindo

Katherine Gotham

Marientina Gotsis

Emma Gowen

Manu Goyal

Elizabeth Grace

Nicci Grace

Patricia Grady-Dominguez

Temple Grandin

Joanna Granich

Cathy Grant

Nicole Grant

William Grant

Doriane Gras

Lara Grau

Carol Gray

Heewon Gray

Kylie Gray

Laura Gray

John Greany

Kirstin Greaves-Lord
Dido Green

GINA GREEN

Jonathan Green

Shulamite Green

Vanessa Green

Rebecca Greenaway

David Greenberg

Rachel Greene

Jennifer Greening

Walter Greenleaf

Jessica Greenlee

Kathryn Greenslade

Jeffrey Gregg

Hojka Gregoric Kumperscak

Ben Gregory

Judith Grether

Whitney Griffin

Gemma Griffith

Sarah Griffiths

Elena Grigorenko

Justin Grill

Emma Grinter

Emma Grisdale

Julius Griskevicius

David Grodberg

JUNE GRODEN

Sabrina Grondhuis

Madeleine Groom

Inge Grootscholten

Steve Grossberg

Jakob Grove

Rachel Grove

Ouriel Grynszpan

Rebecca Grzadzinski

Caroline Guardino

Adam Guastella

MARÍA JOSÉ GUERRA GUIMAREY

Quentin Guillon

Vincent Guinchat

Anna Gulczyńska

Amanda Gulsrud

Serena Gumusoglu

Yağmur Gündüz

Cuihua Guo

Abha Gupta

BABITA GUPTA

Raquel Gur

Emine Gurbuz

Mina Gurevitz

James Gusella

Whitney Guthrie

Anibal Gutierrez

Jenna Gutierrez
Steven Gutstein

Jacalyn Guy

Lisa Guy

Brenda Guzic

Miklos Gyori

April Haas

Kaaren Haas

Mason Haber

Rishika Habib

Bat-Sheva Hadad

Nouchine Hadjikhani

Julie Hadwin

Carl Hagmann

LOUIS HAGOPIAN

Eva-Maria Hahler

Laura Hahn

Cristin Hall

Geoffrey Hall

Alycia Hallady

Anna Eva Hallin

Joachim Hallmayer

Catherine Hambly

Antonia Hamilton

Elizabeth Hammock

Justin Hampton

Lauren Hampton

Sarah Hampton

Narmene Hamsho

Brittany Hand

Benjamin Handen

Jolynn Haney

Gregory Hanley

Mary Hanley

Mary Frances Hanline

Penelope Hannant

Michael Hannon

Blake D. Hansen

John Hansen

Francesca Happe

Sailatul Haque

Ginger Harbin

Amy Harbison

Antonio Hardan

Rebecca Hardiman

Dougal Hare

Abby Hare-Harris

David Harley

Eliza Harley

Madeline Harms

Irene Harmsen

Heather Harrington

John Harrington

Rebecca Harrington 
James Harris

Jennifer Harris

Jill Harris

Ashley Harrison

Sian Harrison-Cross

Clare Harrop

Lynette Hart

Calum Hartley

Sigan Hartley

Catharina Hartman

Kathrin Hartmann

Michal Harty

Daphne Hartzheim

Hannah Harvey

Michael Harwell

Mohammed H. Hassan

Tyler Hassenfeldt

Jan Hastie

Richard Hastings

Megan Hatfield

Aaron Hauptman

Kathryn Hauschild

Alexandra Havdahl

Joshua Haworth

Isobel Hay

Marilyn Hay

Yasmeen Hayat

Stephanie Hayes

Dana Hayward

Eric Hazen

Heather Hazlett

Olive Healy

Pamela Heaton

Marc Hebert

Michèle L. Hébert

Susan Hedges

Darren Hedley

Kevin Heffernan

John Hegarty II

Mikael Heimann

Linda Heitzman-Powell

Gerhard Hellemann

Annika Hellendoorn

Adam Helles

Terhi Helminen

Molly Helt

Heather Henderson

Robert L. Hendren

Charles Henry

Robert Hepach

Susan Hepburn

Catherine Herba

Raine Herbert
Leanna Hernandez

Eugenia Hernandez-Ruiz

Renske Herrema

John Herrington

Irva Hertz-Picciotto

Sean Hess

Eva Hesselmark

Roy Hessels

Dineshani Hettiarachchi

Joe Hettinger

Laura Hewitson

Lynne E. Hewitt

Emily Hickey

Steven Hicks

Elisabeth Hill

Sam Hill

Crystal Hill-Chapman

Ashleigh Hillier

Jennifer Hillman

Claudia L. Hilton

Jeffrey Hine

Alexis Hiniker

Veronica Hinton

Kathrin Hippler

Masahiro Hirai

Shogo Hirata

Tomoya Hirota

Joy Hirsch

Rachel Hirschberger

Tatja Hirvikoski

Fuk Chuen Ho

Helena Ho

Soo Wee Ho

Jessica Hobson

Hannah Hoch

John Hoch

Kevin Hochard

Michal Hochhauser

Marco Hoeksma

Rosa Hoekstra

Ellen Hoffman

Jessica Hoffman

Abigail Hogan

Jeanette Holden

Matthew Hollocks

Jill Hollway

Karla Holmboe

Laura G. Holmes

Mona Holmqvist Olander

Laura Holsen

Rebecca Hommer

Hideo Honda

Emma Honey
Ee Rea Hong

Hwajung Hong

Ickpyo Hong

Michelle Hoogenhout

Jacob Hooker

Stephen Hooper

Daniel Hoover

Serina Hoover

Kathleen Hopf

Ingrid Hopkins

Shari Hopkins

Jamie Horder

Chiara Horlin

ROBERT HORNER

Mady Hornig

Jessica Horst

Massachusetts Hospital

Xiaohui Hou

JANICE HOWARD

Jill Howard

Yamini Howe

Patricia Howlin

Juergan Hoyer

Anne Lise Høyland

Elaine Hsiao

Ching-fen Hsu

Cristiane Hsu

Ju-Wei Hsu

Yulin Hswen

Patty Huan

Chien-Yu Huang

Daniel Hubbard

Caitlin Hudac

Bill J. Hudenko

Rebekah Hudock

Kristelle Hudry

Matthew Hudson

Christopher Hughes

Claire Huijnen

Laura Hull

Kara Hume

Barbara Hummel-Rossi

Neil Humphrey

Karin Humphreys

Rachel Hundley

Ruth Hurst

Bibi Huskens

Nausheen Hussain

Tiffany Hutchins

Ted Hutman

eshayahu Hutzler

Soonjo Hwang

Yoon-Suk Hwang 
Susan Hyman

Yvette Hyter

Marco Iacoboni

Suzannah Iadarola

Dorothea Iannuzzi

GRACE Iarocci

Georgia Iatraki

Lisa Ibanez

Amal Ibrahim

George Ibrahim

Karim Ibrahim

Sally Ibrahim

Kayoko Ichikawa

Valentina Iemmi

Shabnam Iezadi

Chrstine Imms

Brooke Ingersoll

Barry Ingham

Einar T. Ingvarsson

Maria Ioannou

Julie Irish

Dwight Irvin

Julia Irwin

Robert Isenhower

Yuma Ishimoto

Georgi Iskrov

Khaled Ismail

Erkki Isometsa

Jane Iversion

Jana M. Iverson

Jonathan Ives

Michelle Ivey

Jonathan Ivy

Shigeki Iwase

Keise Izuma

Patrick Jachyra

Allison Jack

Douglene Jackson

Elizabeth Jackson

Jeffrey Jackson

Marianne Jackson

Scott Jackson

Suma Jacob

Diane Jacobs

Laudan Jahromi

Mark Jaime

Lorna Jakobson

Monireh jalilil

Jill James

Kathryn Jankowski

Krister Järbrink

Susan Jarmuz-Smith

Anna Järvinen-Pasley
Dasal Jashar

Seye Mohammad Jazayeri

Laurie Jeans

Rachel Jellett

Laura Jelliffe-Pawlowski

ALEXANDER JENSEN

Christina Mohr Jensen

Kathryn Jeter

Jeremy Jewell

Juye Ji

Na Young Ji

Hong Jiang

Yu Jin

Heejoo Jo

Jarle Johannessen

Aesha John

Beth Johnson

Cynthia Johnson

EMMANUEL JOHNSON

Shannon A. Johnson

Ketti Johnson Coffelt

Krista Johnston

Christopher Johnstone

Alice Jones

Christopher Jones

Emily Jones

Garland Jones

Kyle Jones

Leah Jones

Rebecca Jones

Sharon Jones

Ulf Jonsson

Annette Joosten

Dana Jorgensen

Jane E. Joseph

Gagan Joshi

Roger Jou

Amanda Jozkowski

Rubin Jure

Katja Jussila

Amandeep Jutla

jagjeet jutley

Anett Kaale

Aaron Kaat

DUYGU KABA

Louise Kaczmarek

Michael Kaess

Chanaka Kahathuduwa

Ramesh Kaipa

Ann P. Kaiser

Nagaraj Kakanahalli

Layne Kalbfleisch

Zsuzsa Kaldy
Pradip Kamat

Maria Kambouri

Kelly Kamimura-Nishimura

Yoko Kamio

Inge Kamp-Becker

Debra Kamps

Rajesh Kana

Michelle Kandalaft

Daewook Kang

Erin Kang

Aravind Kannan

Stephen Kanne

Sarah Karalunas

Niko Kargas

Claire Karlen

Elizabeth Karp

Christie Karpiak

Jeffrey Karst

Connie Kasari

Wendy Kates

Shiho Kato

Jennifer Katz

Ajay Kaul

Aleksandra Kaurin

Rafal Kawa

Kentaro Kawabe

Sayaka Kawakami

Misa Kayama

Elizabeth Kay-Raining Bird

Yalda Kazemi

veena $\mathrm{KD}$

Brian Keane

Ellen Keane

Chris Keary

Amy Keefer

Brandon Keehn

Rebecca Keehn

Deb Keen

Belinda Keenan

Mickey Keenan

Olumuyiwa Kehinde

Cara Keifer

Ryan Kellems

Elizabeth Kelley

Brian Kelly

Brent Kelsen

Elizabeth Kemeny

Coral Kemp

Steven Kemp

Tal Kenet

Yoed Kenett

Daniel Kennedy

Katrina Kennedy 
Lauren Kenworthy

Nóra Kerekes

Janet Kern

Petra Kern

Connor Morrow Kerns

orly kerub

Mary Louise Kerwin

Klaus Kessler

Leah Ketcheson

Alexandra Key

Rahul Khanna

Rouhallah khara

Ishita Khemka

Neda Khodaverdi

Meena Khowaja

Elizabeth Kiel

Yoriko Kikkawa

Yukiko Kikuchi

Elizabeth Kim

Hyun Uk Kim

Hyung-Goo Kim

Jinah Kim

JinKyung Kim

Lydia Kim

So Hyun Kim

So Yeon Kim

So Yoon Kim

Soo-Jeong Kim

SunKyoung Kim

Sunny Kim

SUNYOUNG KIM

Yael Kimhi

Jessica Kinard

Melissa King

Marcy Kingsbury

Emma Kinnaird

Amanda Kirby

Anne Kirby

Melissa Kirkovski

Bernadette kirkpatrick

Edina Kishonthy

Thomas Kishore

Mikhail Kissine

Jannessa Kitchin

Margaret Kjelgaard

Cheryl Klaiman

Sami Klebanoff

Johan Kleberg

Susan Klein

Daniel Kleinman

Jamie Kleinman

Bonita (Bonnie) Klein-Tasman

Dorit Kliemann
Ami Klin

Katherine Klingensmith

LAURA KLINGER

Mark Klinger

Jessica Klusek

Martin Knapp

Tracy Knaus

Rebecca Knickmeyer

Erin Knight

Victoria Knight

Fiona Knott

John Knutsen

Chia-Tzu Ko

Kenneth Kobak

Steven M. Koch

Robin Kochel

Eva Kočovská

Tiffany Kodak

LYNN KOEGEL

Robert Koegel

Kathy Koenig

Kristie Koenig

Hwan Cui Koh

Gregor Kohls

Kami Koldewyn

Alexander Kolevzon

Judah Koller

Jian Kong

Marianthi Konstantareas

Natalia Kordulewska

Jeffrey Kornitzer

Hirotaka Kosaka

Sezen Kose

Viviane Kostrubiec

Milton Kotelchuck

Amanda Kotolski

Kendra Kotz

Evangelia Kouklari

Evangelia Koukouriki

Hanna Kovshoff

Erin Kraft

Rosy Krajmalnik-Brown

Teri Krakovich

P. Krakowiak

Hilary Kratz

Susan Vaughan Kratz

Nicole L. Kreiser

Jennifer Kremkow

Beate Krieger

Trent Kriete

Ankita Krishnan

Gopee Krishnan

Sheila Krogh-Jespersen
Magdalena Król

William Kronenberger

STEVE KROUPA

S. Jay Kuder

Heather Kuhaneck

Karen Kuhlthau

Jocelyn Kuhn

Marieke Kuiper

Miiamaaria Kujala

Tanu Kukreja

Kristine Kulage

Priya Kumar

Hirokazu Kumazaki

Maithilee Kunda

Shanun Kunnavatana

Jonna Kuntsi

Alice Kuo

HIROSHI KURITA

Jennifer Kurth

Emily Kuschner

Azadeh Kushki

Metehan Kutlu

Kerstin Kutsche

Sanna Kuusikko-Gauffin

Bojana Kuzmanovic

Chantal Labonté

Michael Lafasakis

Fiorenzo Laghi

UTTAMA LAHIRI

Bien Lai

Jonathan Lai

Meng-Chuan Lai

Philip Lai

Wei Wei Lai

Yuen Yi Cynthia LAI

Renee Lajiness-O’Neill

Laurence Lalanne

Kristen Lam

Kristen LaMarca

Liron Lamash

Richard Lamb

Anna Lambrechts

Martine Lamy

Rebecca Landa

Nicole Landi

Oriane Landry

Julia Landsiedel

Alison Lane

Jackie Lane

Justin Lane

Jason Lang

Russell Lang

Chris Lange-Kuettner 
Marc Lanovaz

Kenneth Larsen

Henrik Larsson

Maria Larsson

Alina Lartseva

Robert LaRue

Rinita Laud

Elizabeth Laugeson

Amy Laurent

Margaret Laurie

Marlene Lauritsen

Jenni Lauttia

Blandine Lauzon-Guillain

Evelyn Law

Gloria Law

Miriam Law Smith

Katherine Lawrence

Louise Lawrence

Lauren Lawson

Rebecca Lawson

Daniel J. Laxman

Selena Layden

Thomas L Layton

Ann Le Couteur

Kathy Leadbitter

Geraldline Leader

Justin Leaf

Ronald Leaf

Jocelyn LeBlanc

Linda LeBlanc

Claire Leblond

Eli Lebowitz

Luc Lecavalier

Jennifer Ledford

Brian Lee

Chung eun Lee

Cindy Lee

Clar Y.Q. Lee

Elinda Lee

Evon Lee

Gabrielle Lee

Hoe Lee

Hong Lee

I-Ching Lee

James Lee

Ji Kyung Lee

Jihyun Lee

Keven Lee

Kwangwon Lee

Larry Lee

Leo Lee

Li-Ching Lee

Ya-Chen Lee
SUSAN LEEKAM

Nina Leezenbaum

Kristina Legget

Daniel LeGoff

Fritz-Georg Lehnhardt

Jiedi Lei

Gerry Leisman

Sanne Lemcke

Irene Leo

Hayley Leonard

Helen Leonard

Jean-Francois Lepage

Tuulia Lepisto-Paisley

Jenna Lequia

Paul Lerman

Matthew D. Lerner

Jessica Lester

Cecilia LEUNG

Rachel Leung

Dorit Lev

Anne Geeke Lever

April Levin

Stephen Z Levine

Todd Levine

Adam Lewin

Alan Lewis

Brianna Lewis

Charlotte Lewis

Laura Lewis

Patrick Leytham

Guohua Li

Jun Li

Tingyu Li

Wenfu Li

Xiaoyan Li

Fuxin Lian

Dandan Liang

Hsin-Yi Liang

Ching-Yi Liao

Wei-Ping Liao

Xiaoli Liao

Naomi Libby

Lauren Libero

Klaus Libertus

Melissa Licari

Diane Lickenbrock

Joanne Lim

Nataly Lim

Yi Huey Lim

Sophie Lind

Mark Linden

Olivia Lindly

Ebony Lindor
THOMAS R LINSCHEID

Silke Lipinski

Paul Lipkin

Sarah Lippe

Donna Littlewood

Buyun Liu

Chin-Ting Liu

Emily Liu

Guodong Liu

Janelle Liu

Karen P.Y. Liu

Qiaoyun Liu

Lucy Livingston

Michelle Lizotte

Cecilia Llambias

Elizabeth Llanes

Meghann Lloyd

Ya-yu Lo

DEBRA LOBATO

Jill Locke

Ezra Lockhart

SILVIO LODDO

Rachel Loftin

Sarah Logan

Sheldon Loman

Joanna Lomas Mevers

Michael Lombardo

Antonina Loncarevic

Eric London

Claudio Longobardi

Kristina Lopez

Eva López

David Lopez Perez

Belen Lopez-Perez

Catherine Lord

Catharine Lory

Molly Losh

Amin Lotfizadeh

Eva Loth

Tom Loucas

Soile Loukusa

Anneke Louwerse

Abigail Love

STEVEN LOVE

KATHERINE LOVELAND

Hui Min Low

Milagros Lozano

Leah Lozier

Szu-Ching Lu

Zuhong Lu

Rebecca Lucas

Christina Luckhardt

Joseph Lucyshyn 
Johanna Lüddeckens

Amanda Ludlow

Paul Luelmo

Tove Lugnegard

Ming Lui

Simon Lui

James Luiselli

Colleen Lukens

Emily Lund

Shelley Lund

Brad Lundhahl

Lars-Olov Lundqvist

Yona Lunsk

Yona Lunsky

Caroline Luong-Tran

Rhiannon Luyster

Kristen Lyall

Helena Lydon

Danielle Lyons

Viktoria Lyons

Qing Ma

Tin Shu Terence Ma

Fayez Maajeeny

Suzanne Macari

Jeffrey MacCormack

Megan MacDonald

Mari MacFarland

Samuel Machado

Wendy Machalicek

Dorota Mącik

Kristen Mackenzie

Megan Mackey

Thomas Mackie

Brenna Maddox

Gitte Madsen

Maya Madzharova

Matthew Maenner

Pattie Maes

CEFERINO MAESTU UNTURBE

Lindsay Maffei-Almodovar

Ernesto Magallón-Neri

Sandy Magana

María Magán-Maganto

Daniel Maggin

Iliana Magiati

Christophe Maiano

Kimberly Maich

Simon Maier

Lara Maister

Maria Makrygianni

Evie Malaia

Susan Malcolm-Smith

Anniette Maldonado
Osman Malik

Jarymke Maljaars

Debra Berry Malmberg

Beth Malow

Madhu Mamidala

Oyku Mance Calisir

Gregory Mancil

David Mandell

William Mandy

Christopher Manente

Joseph Manfredonia

Shanley Mangeot

Karthik Mani

Catherine Manning

Patricia Manning-Courtney

khalid mansour

DIPALI MANTRY

Stacy Manwaring

Jorge Manzo

Katie Maras

Serge Marchand

Loredana Marchica

Elysa Marco

Lee M. Marcus

Lucia Margari

Francesco Margoni

Carla Marin

Theodoros Marinis

Maria Marinopoulou

Marlina Marlina

Carla Marquez

Becky Mars

Christina Marsack-Topolewski

Peter Marschik

Rachel Marsden

Lauren Marsh

Alena Marshall

Jeanne Marshall

Andres Martin

Caren Martin

Gary Martin

Lee Martin

Peter Martin

Luis Martín-Antón

Joelle Martineau

Jose Martinez

Raquel Martinez

Frances Martinez-Pedraza

Maria Martzoukou

Helen Marwick

David Mason

Lee Mason

Wolfgang Mastnak
Maya Matheis

Danielle Mathersul

Heath Matheson

Binoy Mathew K V

Johnny Matson

Soichiro Matsuda

Nicole Matthews

Marja-Leena Mattila

Whitney Mattson

Andrea Maughan

Seyedmohammad Mavadati

Chris May

Richard May

Murray Maybery

Melissa Maye

Jennifer Mayer

Susan Dickerson Mayes

Hajar Mazahery

Carla Mazefsky

Michel Maziade

Micah Mazurek

Greta Mazzaggio

Phil McAleer

Grainne McAlonan

Tomomi McAuliffe

Allyssa McCabe

Erin McCanlies

Leigh McCann

Patricia McCartney

Lindsay McCary

Mark McCaulley

Maryellen McClain

Joseph McCleery

Mary McCollum

Helen McConachie

Beth McCormick

Carolyn McCormick

Stephanie McCoy

Adam McCrimmon

Eamon McCrory

Matthew McCrudden

Erin McCurdy

Jena McDaniel

Christin McDonald

Nicole McDonald

T A McDonald

Christina McDonnell

CHRISTOPHER MCDOUGLE

Andrea McDuffie

Jennifer McFarland-Whisman

Tyler McFayden

Elizabeth McGhee Hassrick

Margaret McGonigle-Chalmers 
John H. McGrew

Kelly McGuire

Laura Lee McIntyre

Nancy McIntyre

Elizabeth McKenney

Elizabeth McKernan

Bethany McKissick

Sharon McLaughlin

Tara McLaughlin

Laurie McLay

Scott McLeod

Camilla McMahon

Chris McManus

Stephen Edward McMillin

Carly McMorris

Cheryl McNeil

Jordan McNeill

James McPartland

Melissa McPheeters

Rebecca McStay

Alana McVey

bikash medhi

Lisa Meeks

Sunil Mehta

James Meindl

Kerstin Meints

A. Melinder

Catherine Mello

Claudia Mello

Britany Melton

Megan Melton

AmirHossein Memari

Idan Menashe

Maarten Mennes

Laura Ment

Eduardo Mercado

Hannah Merrick

CAROLYN Mervis

Elizabeth Meshes

Gary Mesibov

Daniel Messinger

Allison Meyer

Echo Meyer

Suzanne Michaels

Jacob Michaelson

Frank Middleton

Judith H. Miles

Andrew Miller

Arnold Miller

Carol Miller

David Miller

Fiona Miller

Haylie L. Miller
John Miller

Judy Miller

Meghan Miller

Veronica Miller

Julie Miller-Hernandez

Alyssa Milot

Thomas Milson

Raymond Miltenberger

Nicholas Minar

Beth Mineo

Camelia Minica

Ilaria Minio-Paluello

Mendy Minjarez

Noha Minshawi

Nancy Minshew

Kojo Mintah

Joseph Mintz

Ana Miranda

Sarah Mire

PAT MIRENDA

Maliheh Miri

Lisa Mische Lawson

Avinash Mishra

Eric Mitchell

peter mitchell

Yoshihiro Miyake

Amirhossein Modabbernia

Yogeswara Modugumudi

Maria Mody

Jeremy Moeller

Anna Moffat

Melanie Mogavero

Fateme Mohammadi

Gomathi Mohan

Nina Molendijk

Sophie Molholm

DANIEL MOLINA

Jorge Molina-López

Monica Molinaro

Elizabeth Mongillo

Farhad Montazeri

Charlotte Montgomery

Janine Montgomery

Cecilia Montiel-Nava

Eric Moody

Dennis Moore

Jessica Moore

Timothy Moore

Mariana Morales-Chávez

Mariela Morelli

Daniel Moreno-De-Luca

Laura Morett

Lindee Morgan
Alain Morin

Hiroyuki Moriuchi

Michael Morrier

Laren Morris

Rae Morris

Sonia Morris

Susan Morris

Kerrianne Morrison

Matthew Mosconi

Rachel Moseley

Lauren Moskowitz

Maya Mosner

Joanna Moss

Karin Mössler

Stewart Mostofsky

sara motamed

Laurent Mottron

Douglas Moul

Emily Moulton

Svend Erik Mouridsen

Sabine Mous

Ahmadreza Movahedi

Tinneke Moyson

Beth Mozolic-Staunton

Alicia Mrachko

Daniel W. Mruzek

Joshua Muggleton

Faraz Mughal

Reem Muharib

Rebecca Muhle

nahit mukaddes

Sharmila Mukherjee

Cari-lene Mul

Surabhi Mulchandani

Christina Mulé

Mathias Müller

Ralph-Axel Müller

Keriim Munir

Jeff Munson

Jeffrey Munson

Filippo Muratori

Michael Murias

Eric Murphy

Treasa Murphy

Aja Murray

Donna Murray

Scott Murray

Kimberly Murza

Rachael Muscatello

Raghad Mutabbakani

Anne Muth

Scott Myers

Jalilevand N 
Fabienne Naber

Anne-Marie Nader

Rami Nader

Aparna Nadig

Marisa Nagano

Allison Nahmias

Letitia Naigles

Mariko Nakanishi

Nozomi Naoi

adam naples

Jayanthi Narayan

Naoko Narita

Ramzi Nasir

Kate Nation

Andre Ndobo

Rebecca Neal-Beevers

Cameron Neece

Kristina Neely

Leslie Neely

Lisa Negri

Travis Nelson

Wei Siong Neo

Kirsten Ness

Emily Neuhaus

Ann Neumeyer

Rose Nevill

Cynthia Nevison

Nigel Newbutt

Mindy Newhouse-Oisten

Craig Newschaffer

Deborah Newton

Anh Kiet Danny Nguyen

Kien Nguyen

Lam-son Nguyen

David Nicholas

Chad Nichols

Toby Nicholson

Robert Nickel

Christina Nicolaidis

Humberto Nicolini

Alexander Niculescu III

Emma Nielsen

Mark Nielsen

Annabel Nijhof

Lena Niklasson

Christos Nikopoulos

firoozeh nilchian

Jennifer Ninci

Adam Noah

Anna Nobili

Jean Paul Noel

David Noelle

Ilse Noens
Cortenay Norbury

Courtenay Norbury

Christine Nordahl

Anders Nordahl-Hansen

Fritjof Norrelgen

Jessie Northrup

Laura Nota

Nikola Nowack

Kerri Nowell

Sara Nowell

Erika Nurmi

Heather Nuske

Amy K. Nuttall

Cheryl Nye

Johan Nyrenius

Lindsay Oberman

Justin O'Brien

Iva Obrusnikova

Julia O'Connor

Karen O'Connor

Mary O'Connor

Christina Odeh

Samuel L. Odom

Anoek Oerlemans

Christine Ogilvie

Billy T Ogletree

Marguerite O'Haire

Roderick O'Handley

Manabu Oi

Roald Øien

Yuko Okamoto

Meral Çilem Ökcün Akçamuş

Emily Olfson

Melissa Olive

Patricia Oliveira Lima Muñoz

Chris Oliver

Kelsey Oliver

Rafael Oliveras-Rentas

Thomas H. Ollendick

Stephen Oller

Eric Olofson

Lise Olsen

Linda O'Neill

Elizabeth O'Nions

Iris Oosterling

Devon Oosting

Folakemi Oredugba

Alyssa Orinstein

Jeff Oristaglio

Sarah Ormond

Gael Orsmond

Mohammad Majid Oryadi-Zanjani

Hirokazu Osada
Christopher Osborn

Lisa Osborne

Albert Osei

Soren Ostergaard

Katrina Ostmeyer

Carmel O'Sullivan

Karl Oswald

Donald Oswald (Do not invite)

Sadao Otsuka

Katrin Ounap

Opal Ousley

Terry Overton

Lluis Oviedo

Selda Ozdemir

Sirin Ozdemir Tapan

Heval Ozgen

Sally Ozonoff

Ann Ozsivadjian

Lisa Paar

Ciara Padden

Cherie' Page

Pamela Pallett

Mark J. Palmieri

Orobal Paloma

Ruben Palomo

Michelle Palumbo

C Y Pan

Chien-Yu Pan

Maria Panagiotidi

Heather Panczykowski

Vincent Pandolfi

Simonetta Panerai

Elizabeth Pang

Melissa Pangelinan

Robin Panneton

Nicole Papadopoulos

Vaya Papageorgiou

Despina Papoudi

Tejas Parekh

Chandni Parikh

Susan Parish

Julia Parish-Morris

Hye Ran Park

Jung Eun Jeanne Seyeon Park

Mi N Park

Mina Park

Krystal Parker

Susan Parks

Valentina Parma

Jeremy Parr

Dave Parsons

Lauren Parsons

Sandra D. Parsons 
Sarah Parsons

Thomas Parsons

Elise Pas

Olivier Pascalis

Greg Pasco

Alvaro Pascual-Leone

Sarah Paterson

Michelle Patriquin

RHEA PAUL

Markus Paulus

Nalin Payakachat

Jonathan Payne

Jessica Paynter

Georgina Peacock

Amanda Pearl

Jamie Pearson

Laura Pecora

Meredith Pecukonis

Ernest Pedapati

Kahsi Pedersen

Melanie Pellecchia

Elizabeth Pellicano

Kevin A. Pelphrey, Ph.D

Morgan Peltier

Mirabel Pelton

Catherine Pemble

Yuwen Peng

Melanie Penner

Robert C. Pennington

Debra Pepler

Katherine Perdue

Michael Peres de Medeiros

Georgina Perez Liz

Patricia Pérez-Fuster

Margaret Pericak-Vance

James M. Perrin

Julia Perrotta

LYNN PERRY

Antonio Persico

B. Peters

Jessica Petri

Anna Petursdottir

Rebecca Pfeffer

Beth Pfeiffer

Gerit Pfuhl

Janice Phung

Rosalind Picard

Giorgia Picci

Katherine Pickard

Andrew Pickles

Karen Pierce

Nigel Pierce

Mirjam Pijl
Judith Pijnacker

Victoria Pile

Dhanya Pillai

Jaime Pineda

Amy E. Pinkham

JA Pinto-Martin

pascale piolino

Daniele Piscitelli

Ewa Pisula

Kenneth Pitetti

Naomi Pitskel

V. HOlly Pitt

Keenan Pituch

Anna Piwowarczyk

Anne Pizzano

Aske Plaat

Pascale PLANCHE

Joshua Plavnick

Anja Plemenitas

Daniela Plesa

Bertram Oliver Ploog

Allison M. Plumb

Lindsay Pocock

Jennifer Pokorny

Elizabeth Pokorski

Alan Poling

Laura Politte

Lara Polse

Milena Pondé

Daniel Poole

Jennifer Poole

Svetlana Popovic

Ben Popple

John Port

David Posey

Irina Poslawsky

Daniel Posner

Stephen Post

Jessica Postil

Marie-Christine Potvin

Renee Poulin

Fritz Poustka

Kelly Powell

Kristen Powers

Michael D. Powers

Benjamin Praker

Paola Prandini

Patricia Prelock

Paula Prendeville

Alison Presmanes Hill

Clare Press

Jonathan Preston

GAVIN PRICE
Molly Prigge

Julia Proft

Beth Provost

Debra Prykanowski

Daisy Pua

Cara Pugliese

Nicolaas Puts

Zheala Qayyum

Xueqin Qian

Uvais Qidwai

Constance Dean Qualls

Thomas Quatieri

Jean Quigley

Kathleen A. Quill

Leah Quinlivan

Eve-Marie Quintin

Linda Quirmbach

Adam Qureshi

Anjali R

Aspasia Stacey Rabba

Shantha Radhakrishna

Eugenia Radulescu

Ebrahim Rahbar Karbasdehi

A. M. Pervez Rahim

M. raichle

Ramesh Rajan

SATHYABAMA RAMACHANDRAM

Rebecca Ramnauth

Sobhana Ranjan

James Rankin

Shripada Rao

Melissa Raspa

Jessica Rast

Eva Ratai

Belinda Ratcliffe

Nann Ratner

Allison Ratto

Caitlin Ravichandran

Dora Raymaker

Judy Reaven

Elizabeth Redcay

Linda Reddy

Vasudevi Reddy

Alexandra Reed

Catherine Reed

Phil Reed

Clare Reeder

R. Matthew Reese

Christine Reeve

Avi Reichenberg

Brian Reichow

Angela Reiersen

Marian Reiff 
Bryan Reimer

Vanessa Reinhardt

Debra Reinhartsen

Debra Reisinger

Whitney Relf

Amanda Rendall

Patricia Renno

Leslie Rescorla

Stephanie Reszka

Alessandra Retico

Nuri Reyes

Stacey Reynolds

Jessica Ribeiro

Deborah Riby

Cynthia Riccio

Paul Riccomini

Catherine Rice

Caroline Richards

Gareth Richards

Amanda Richdale

Nick Riches

David Richman

Andreas Riedel

Robert Rieske

Sarah Rieth

Nicole J. Rinehart

Alexander Ring

Joel Ringdahl

Shannon Ringenbach

Kristin Rispoli

Mandy Rispoli

Valentina Riva

mélina rivard

Giacomo Rizzolatti

Cristine Roberts

Jacqueline Roberts

Jane Roberts

Sarah Roberts

Timothy Roberts

Diana Robins

Janine Robinson

Sally Robinson

Andrew Robson

Naomi Rodas

Jacqui Rodgers

Jonathan Rodgers

Geovanna Rodriguez

Jairo Rodríguez-Medina

Amanda Roestorf

Herbert Roeyers

Cynthia Rogers

Kimberley Rogers

Sally J. Rogers
Donald Rojas

Pamela Rollins

RAYMOND ROMANCZYK

Claudia Romano

Nanda Rommelse

Mary Ann Romski

Jenny Root

Danielle Ropar

Chad Rose

Destanie Rose

Shannon Rose

Tamara Rosen

David Rosenberg

Rebecca Rosenberg

Miriam Rosenberg-Lee

Gabriela Rosenblack

Gabriela Rosenblau

Denise Ross

Peter Ross

Veerle Ross

Daniel Rossignol

Erin Rotheram-Fuller

Anne Roux

Justin P. Rowberry

Meagan Roy

Rachel Royston

Agata Rozga

Eric Rubenstein

Julie Rubin

Lisa Ruble

Douglas Ruderfer

Alokananda Rudra

Victoria Russ

Sarah Russell

Natalie Russo

Theresa Russo

Nicole Russo-Ponsaran

Liliana Ruta

Helena Rutherford

M.D. Rutherford

Marion Rutherford

Ellen Rutt

Michael Rutter

Laura Ruzzano

Agnieszka Rynkiewicz

Khaled Saad

Mohammad Nasser Saadatzi

Maura Sabatos-DeVito

Aderbal Sabra

Filipa Sa-Carneiro

Lori-Ann Sacrey

Ghasem Sadeghi Bajestani

Amy Sadek
Evald Saemundsen

Rachel Saffo

Beth Saggers

Catherine Saint-Georges

Aya Saito

Ali-Akbar Salari

Alisha Salerno

Siti Salwah Salim

Erica Salomone

Ann Sam

Andrea Samson

Sela Sanberg

Patricia Sanchez Lizardi

Ana Sanchez-Garcia

Micheal Sandbank

Jessica Sanders

Cheryl Sandford

Sven Sandin

Pooja Sani

Elise Sannar

Stephanie Sansone

Frank Sansosti

Damian Santomauro

Mariline Santos

Tonja Sappok

Tewarit Sarachana

Maria Sarafidou

Zoe Sargent

Esra Sarigecili

Sepideh Saroukhani

Encarnación Sarria

Dustin Sarver

Tsukasa Sasaki

Noah Sasson

Kari Sassu

Celine Saulnier

Barbara Saunders

Oliver Saunders Wilder

Melissa Savage

Ralph Savarese

Richa Saxena

Lawrence Scahill

Angela Scarpa

Maria Luisa Scattoni

G. Bradley Schaefer

Dorothy Schafer

Rik Schalbroeck

Carol Schall

Ulrich Schaller

George Schanding

Anke Scheeren

Gretchen Scheibel

Robert Scheidt 
Mindy Scheithauer

Stefanie Schelinski

Michaela Schenkelberg

Stephen Scherer

Suzanne Scherf

Hannah Schertz

LA Schieve

Hillary Schiltz

Synnve Schjolberg

Wim A.J.M. Schlooz

R.W. Schlosser

Ellyn Schmidt

Rebecca Schmidt

Lauren Schmitt

Christina Schmitz

Andrea Schneider

Ben Schneider

Sarah Schoen

Alison Schonwald

KIMBERLY A SCHRECK

LAURA SCHREIBMAN

Bastian Schrott

Manuela Schuetze

Robert Schultz

Sarah Schultz

Cynthia Schumann

Claire Schutte

Jessica Schuttler

Tobias Schuwerk

Emmanuel Schwab

Lizaan Schwartz

Benjamin Schwartzman

José Schwartzman

AJ Schwichtenberg

Lynette Scotese-Wojtila

Jessica Scott

Melissa Scott

Billie Searing

Matthew Segall

Małgorzata Sekułowicz

Marsha Seltzer

Angel Selvaraj

Stephanie Seneff

Hatice Senel

Elif Funda Sener

Atsushi Senju

Amie Senland

Marike Serra

ceyhun servi

HyeKyeung Seung

Javier Sevilla

Monique Seymour

Robin Shafer
Rebecca Shaffer

Keith Shafritz

Punit Shah

Vaishal Shah

Erik Shank

Benjamin Shapiro

Ashish Sharma

Shivani Sharma

William Sharp

Christopher Sharpley

Khulood Shattnawi

Paul Shattuck

Robert Shavelle

Lindsay Shea

Stephen J. Sheinkopf

R Sheldrick

Janie Shelton

Yiping Shen

Jay Shendure

Elizabeth Shephard

Daniel Shepherd

Collin Shepley

Elizabeth Sheppard

Kelly Sheppard

Elliott Sherr

Michele Sherwin

Bhavin Sheth

Wendian Shi

Yang Shi

Frederick Shic

Aaron Shield

N. Shields

Andy Shih

Amelle Shillington

Anya Shindler

Stephanie Shire

Carolyn Shivers

Seoyoung Shon

Stephen Shore

Elizabeth Shpphard

Oren Shtayermman

Lauren Shuffrey

Kathy Shum

Jordan Shurr

Panagiotis SIAPERAS

Bryna Siegel

Matthew Siegel

Darryn Sikora

Michael Siller

Catarina Silva

Deborah Silver

Jill Silverman

Laura Silverman
Jessica Simacek

David Simmons

Grace Simmons

Eileen Simon

Nicole Simon

Emily Simonoff

Kate Simpson

RICHARD L SIMPSON

Charlotte Sinding

Alison Singer

Harvey Singer

Lauren Singer

Jennifer Singh

NIRBHAY SINGH

Nidhi Singhal

Judith Sinzig

Joshua Skewes

Daniel Skorich

Pernille Skovbo Rasmussen

Jeff Skowronek

Simona Skripkauskaite

David Skuse

Elspeth Slayter

Vicky Slonims

LaRene Smart

A Smerbeck

Aslyn Smith

Brenda Smith

Caroline Smith

Cary stacy Smith

Catherine Smith

Chris Smith

Elizabeth Smith

Isaac Smith

Isabel Smith

Matthew Smith

Rachel Smith

Tristram Smith

Sinéad Smyth

Cheong Sng

Ryuhei So

Neelkamal Soares

Kate Sofronoff

Gnakub Soke

Marcus Sokolowski

canan sola özgüç

Marjorie Solomon

Shrikari Somashekar Rao

Massimiliano Sommantico

Ranran Song

Yiqi Song

Yongning Song

Latha Soorya 
Marlene Sotelo

Igor Sotgiu

Timothy Soto

Mohammad Saber Sotoodeh

Isabelle Soulières

Mikle South

Sophie Sowden

Debbie Spain

Marisa Spann

Laura Sparaci

Janet Spector

Vicki Spector

Antoinette Spek

Sara Spencer

Trina Spencer

Laurie Sperry

Michael Spezio

Donna Spiker

Fred Spooner

Amy D. Spriggs

Shylaja Srinivasan

Wouter Staal

Alexia Stack

Nicole Stadnick

Steven Stagg

AUBYN STAHMER

Catherine Stamoulis

Aglaia Stampoltzis

Heidi Stanish

Cedomir Stanojevic

Jennifer Stapel-Wax

Kerri Staples

Elizabeth Starr

Fabrizio Stasolla

Johannas Stauder

Ekaterini Stavrou

Shelly Steele

Mandy Steiman

Leah I. Stein Duker

Nina Stenberg

Donald Stenhoff

Kevin Stephenson

Alexandra Stergiou

Paula Sterkenburg

Audra Sterling

Yael Stern

Laura Sterponi

Paul Sterzing

Hanna Stevens

Bruce Stevenson

Jennifer Stevenson

Roger Stevenson

Ryan Stevenson
Hugh Stewart

Mary Stewart

Janine Stichter

Andrew Stickley

Anja Stiller

John Stins

Kevin Stoddart

Mark Stokes

Michael Stolte

WENDY STONE

Laura Stoppelbein

Eric Storch

Michael Storz

Alicia Strain

John Strang

Elizabeth Stratis

Kristin Strauss

Mark Strauss

Dorothy Strickland

Sheri Stronach

Kristen Strong

Sandra Strunz

Dominik Strzałka

Breanna Studenka

Kara M. Styck

$\mathrm{Yi}$ (Esther) $\mathrm{Su}$

Michelle Suarez

Adele Suda

Joyce Suh

Chonlaphat Sukasem

Denis Sukhodolsky

Rhylee Sulek

Renee Sullivan

Chika Sumiyoshi

Jennifer Sumner

Vaithiamanithi Sundaram

Connie Sung

Kyongje Sung

Min Sung

Jennifer Suppo

Suprajitno Suprajitno

Suma Suswaram

Clare Sutherland

Katsuaki Suzuki

Tomoko Suzuki

Hanna Swaab

Deanna Swain

Amy Swanson

Geoffrey Swanson

Meghan Swanson

Thayne Sweeten

John Swettenham

Naomi Swiezy
Lauren Swineford

Jennifer B. G. Symon

FRANK SYMONS

CHRISTINE SYRIOPOULOU

Peter Szatmari

Christen Szymanski

Carmen Tabares

Katsuhiko Tabuchi

Stefano Taddei

Helen Tager-Flusberg

Mohammad Taheri

Hamidreza Taheri-Torbati

Tetsuya Takahashi

Yukari Takarae

Toshinobu Takeda

Meagan Talbott

Nicole Talge

Leanne Tamm

Beron Tan

Hiroki Tanaka

Daniel J. Tancredi

Nidhi Taneja

Julia Tang

Karen Tang

Masako Taniike

Amy Tanner

Jonathan Tarbox

Balazs Tarnai

Teresa Tavesoli

Briana Taylor

Bridget Taylor

Janelle Taylor

Jerome Taylor

Julie Taylor

Lauren Taylor

Margot Taylor

Mami Tazoe

Wolfgang Teder-Salejarvi

LAVINIA TEIXEIRA-MACHADO

Saime Tek

Cassandra Tellegen

Valerie Temple

Kayla Ten Eycke

Leontine ten Hoopen

Elena Tenenbaum

Jing Xin Teo

Arhonto Terzi

Charles Tessier

Hanna Thaler

Eleni Angeliki Theodosi

Ron Thibert

Jane Thierfeld Brown

Robyn Thom 
Benjamin Thomas

Molly Thomason

Marcus Thomeer

Barbara Thompson

Grace Thompson

Einar Thorsteinsson

Emily Thrower

Matthew Thullen

Audrey Thurm

Anna-Maija Tiainen

Adrienne Tierney

Laura Timonen-Soivio

Leigh Tipton

Maria Tirado

Durgesh Tiwari

Shivani Tiwari

Tomas Tjus

Carol To

J. Tobias

Megan Tobin

Theodore Tomeny

BRUCE TONGE

Cetin TOPUZ

Sylvie Tordjman

Nina Torheim Thronæs

Karen Toth

Vasiliki Totsika

Luciana Tovo-Rodrigues

JEANNE TOWNSEND

Brittany Travers

Jason Travers

Jenna Traynor

Noémie Treichel

David Trembath

Isabelle Tremblay

Jayne Trickett

Emily Trimmer

Eva Trinh

Vanessa Troiani

Giovanni Troiano

Daniela Tropea

Eva Troyb

Chia-Liang Tsai

Hsiao-Wei Tsai

Luke Y. Tsai

Sandra Tsang

Tawny Tsang

Ling Tsao

Choi Yeung Andy TSE

Angela Tseng

FOTINI TSIFTZI

Ioanna Tsiouri

Kenji Tsuchiya
Roberto Tuchman

Ray Tucker

Megan Tudor

Christopher Tullis

Birkan Tunc

Jack Turban

Paul Turcotte

Gustavo Turecki

Marco Turi

Jeremy Turk

Pauline Turnbull

Karen Turner

Lauren Turner-Brown

Nicole Turygin

Miriam Twomey

Lucina Uddin

Miko Uljarevic

Ganapathy Umaiorubagam

Ayumi Umino

Emory University

Shota Uono

Maria Urbano

Anna Urbanowicz

David Vagni

Eleni Vailiopoulou

Vivek Vajaratkar

Giulio Valagussa

Maria Valdovinos

Marco Valenti

Maria Valicenti-McDermott

Marit van Bakel

Ingrid van Balkom

Ina A. van Berckelaer-Onnes

Mary Van Bourgondien

Emma van ****Daalen

Judy Van de Water

Marianne van den Bree

Stephanie Van der Donck

Rutger van der Gaag

Ruth Van der Hallen

Anna van der Miesen

Agnies Van Eeghen

Lien Van Eylen

Kees van Heeringen

Valérie Van Hees

Toni Van Laarhoven

Kim Van Orden

Samuel van Rensburg

Eeske van Roekel

Jan van Santen

Gerrit Van Schalkwyk

Francisca van steensel

Daphne van Steijn
Bob van Tiel

Patricia van Wijngaarden-Cremers

Ernst VanBergeijk

Steven Vanmarcke

Jennifer Vannest

Marleen Vanvuchelen

Ravi Prasad Varma

Roma Vasa

Ivana Vasilevska Petrovska

Amy Vaughan Van Hecke

Jorge Vazquez

Olivia Veatch

Svetla Velikova

Milen Velinov

Aldina Venerosi

Edy Veneziano

Pamela Ventola

Lisette Verhoeven

Frank Verhulst

Peter Vermeulen

Angelina Vernetti

David Vernon

Ty Vernon

Stefano Vicari

Sara Victor

Susanna Villa

Laurie Vismara

Ingela Visuri

Giacomo Vivanti

Paola Vizziello

Vanessa Vogan

Joanne Volden

Melek Volkan-Yazici

Martha Volker

Martin Volker

Timothy Vollmer

Pamela von Hurst

Avery Voos

Helen Vuong

David Wachob

Lee Wachtel

David Wacker

Hannah Waddington

Joshua W Wade

Shannon Wagner

Allison Wainer

Jane Waite

Einat Waizbard-Bartov

Akio Wakabayashi

Małgorzata Walęcka

Cheryl Walker

Dennis Wall

Greg Wallace 
Casey Walsh

Christopher Walsh

Cian Walsh

Reubs Walsh

Catia Walter

Sasha Walters

Katherine Walton

Ming Wai Wan

Chuanpo Wang

Chun-Hou Wang

Jia-En Wang

Paul Wang

Qiandong Wang

Quan Wang

Zheng Wang

Sandra Ward

Marji Erickson Warfield

Rosemary Waring

Katherine Warnell

Stephen Warren

Zachary Warren

Petra Warreyn

Patricia Waters

Laci Watkins

Renee Watling

Linda R. Watson

John Wattam-Bell

Jessica Waxler

Marketa Wayhelova

Priscilla Weaver

Natalie J. Webb

Sara Webb

Amanda A. Webster

Hua Wei

Paige Weinger

Emma Weisblatt

Omri Weisman

Deborah Weiss

Jonathan Weiss

Lauren Weiss

MARY J WEISS

Amy Weitlauf

Carol Weitzman

Sharon Welby

Henry Wellman

Laurie Wellner

Oliver Wendt

Pei-Lin Weng

Elisabet Wentz

Kelsey West

Marleen Westerveld

Alexander Westphal

Heather Westwood
Amy M. Wetherby

Kelly Whalon

Anne Wheeler

Barbara Wheeler

Sally Wheelwright

Lydia Whitaker

Barbara White

Catriona White

Sarah White

Susan White

Andrew J. O. Whitehouse

Daniel Whitney

Rondalyn Whitney

Holly Whittenburg

Lisa Whittingham

Caroline Whyatt

Elisabeth Whyte

Bruno Wicker

Andrea Wieckowski

Lisa Wiggins

Lieke AMW Wijnhoven

Ellen Wijsman

JULIA WILBARGER

Lucy Wilde

Preston Wiles

Elizabeth Will

A Willems

Diane Williams

Justin Williams

Kyle Williams

Marian Williams

Natalie Williams

Tracey Williams

Zachary Williams

Howard Wills

Alexander Wilson

C. Ellie Wilson

Charlotte Wilson

Kaitlyn Wilson

Gayle C. Windham

Logan Wink

Adam Winsler

Harland Winter

Kat Witt

Gail Wolf

Julie M. Wolf

Lorraine Wolf

Rachel Wolf

Jason Wolff

Jeanne Wolstencroft

Connie Wong

Jasin Wong

Virginia Wong
Nina Wong Sarver

Charles Wood

Jeffrey Wood

Kate Woodcock

Ashley Woodman

Susan woolfenden

Rachel Worthington

Tiffany Woynaroski

Janet Wozniak

Kristyn Wright

Pam Wrigley

Ching-lin Wu

Wei-Li Wu

Yanhong Wu

Fagen Xie

$\mathrm{Ke} \mathrm{Xu}$

Yaoying $\mathrm{Xu}$

Gulnoza Yakubova

Jie Yang

Yung-Jui (Daniel) Yang

Vincent Yau

Yanki Yazgan

Gozde Yazkan Akgul

Tamara Yee

Hsiaoju Yen

Benjamin Yerys

Michael K. Yeung

Huso Yi

İlker Yilmaz

Larry Yin

Marissa Yingling

Nurit Yirmiya

Paul Yoder

Robert Yolken

J. Yoo

wn-ho Yoon

Isabel Yorke

Larry Young

Robyn Young

Eric Youngstrom

Luodi Yu

Timothy Yu

Serife Yucesoy-Ozkan

Kunio Yui

Nicola Yuill

Najah Zaaeed

Benjamin Zablotsky

Ditza Zachor

Hasan Zaghlawan

Mati Zakai-Mashiach

Rano Zakirova Engstrand

Tiziana Zalla

irina zamora 
Emily Zane

Kathleen Zanolli

Gemma Zantinge

Jennifer Zarcone

Maryam Zare Jeddi

Sasha Zeedyk

Waganesh Zeleke

ousseny zerbo

Cuilin Zhang

Ling Zhang

Xin Zhang yumin zhang

Huaiyang zhao

Jing Zhao

Shuting Zheng

Zhichun Zhou

Gaoxia Zhu

Michelle Zimmer

Andrew Zimmerman

Kathleen Zimmerman

Alexandra Zinck

Giada Zoccatelli
Tiago Zortea

Rachael Zubal-Ruggieri

Rosa Zuccarello

Kenneth Zucker

Katharine Zuckerman

Nicole Zurcher

Lonnie Zwaigenbaum

Fenny Zwart

Mark Zylka

Publisher's Note Springer Nature remains neutral with regard to jurisdictional claims in published maps and institutional affiliations. 